\title{
Analysis and Prediction of Electrical Contact Resistance for Anisotropic Conductive Adhesives
}

\author{
Aleš Duraj ${ }^{1)}$, Pavel Mach ${ }^{1)}$ \\ ${ }^{1)}$ Department of Electrotechnology, Faculty of Electrical Engineering \\ Czech Technical University, Prague 6, 166 27, Czech Republic \\ E-mail: duraja@centrum.cz
}

\begin{abstract}
Anisotropic conductive adhesives (ACAs) and anisotropic conductive films (ACFs) are promising solder alternatives. These materials are preferable for fine pitch and ultra-fine pitch connections in electronics assembly. Quality of these interconnections is usually evaluated with measurement of electrical resistance. This paper describes few simple models for prediction of electrical contact resistance in ACAs interconnects. Nevertheless in these models is usually neglected effect of thin insulating layer between conductive particles and conductive tracks on substrate. Therefore two different methods for evaluation of electron tunneling resistance are analyzed and implement into models for prediction of electrical contact resistance of ACAs/ACFs. Extended models are directly compared with experimental results. Also effect of bonding pressure and different materials of conductive tracks is analyzed.
\end{abstract}

\section{INTRODUCTION}

Lead-free soldering is extensively used for interconnections in electronic assembly. Solder joints serve good mechanical, thermal and electrical interconnections. Nevertheless electrically conductive adhesives (ECAs) are also used as an ecological replacement for a tin-lead soldering. ECAs were developed especially for joining in applications where the use of high temperature (necessary for soldering) is impossible. Also special isotropic conductive adhesives (ICAs) are used for attaching large area printed circuit boards or LTCC to thick metal ground planes in various microwave applications. Special types of ECAs - anisotropic conductive adhesives (ACAs) and anisotropic conductive films (ACFs) are mainly used in flip-chip assembly, chip-on-film (COF), chip-on-glass (COG) or for flexible circuit assembly applications (chip-on-flex).

ACAs and ACFs are composite materials which consist of spherical conductive particles dispersed uniformly in an insulating resin matrix that possesses adhesive properties. The basic material of the matrix is usually thermosetting epoxy resin and it is expected to provide stable adhesion between the two components and also electrical insulation where necessary. Electrical conduction between the chip and substrate is achieved through the conductive particles trapped between the corresponding conductive tracks on chip and substrate when pressure and heat are applied simultaneously. These particles are usually balls with conductive layer (polymer $+\mathrm{Au}, \mathrm{Ni}+\mathrm{Au}$ ). These particles are squeezed between electrodes during actual bonding and this deformation increases the contact area. It lowers the contact resistance and improves quality of interconnections. Therefore quality of these connections is usually evaluated with measurement of electrical resistance. Joining with ACAs and ACFs is very fast and successfully spreading technology because of there is also no requirement of applying additional underfill.

For small scale applications and high frequencies is also necessary prepare predictions of electrical contact resistance. In the recent past, a number of models have been developed for understanding of these phenomena. However, as this paper will show, the values of electrical contact resistance predicted by these models are in significant disagreement with each other and with values measured experimentally, which are much higher. It is due to many simplifications in these models. Especially neglect of material roughness and electron tunneling has radical effect on electrical contact resistance. This paper presents two different methods for evaluation of electron tunneling resistance. These evaluations are implemented into prediction of electrical contact resistance in ACAs/ACFs 
interconnects. Extended models for electrical resistance prediction are directly compared with experimental results.

\section{CONTACT RESISTANCE MODELING}

Standard procedure for modeling of electrical contact resistance between particles and conductive pads is accomplished in two steps:

1) determination of contact area $A$ versus bonding force $F$

2) determination of contact resistance $R_{C}$ versus contact area $A$

For evaluation of contact area $A$ are usually used numerical methods or basic Hertzian theory:

$$
\begin{aligned}
& A=\pi \cdot a^{2} \\
& a=\sqrt[3]{\frac{3 F d}{8 E^{*}}}
\end{aligned}
$$

where $a$ is a contact radius, $F$ is bonding force, $d$ is a diameter of particle, $E^{*}$ is the effective elastic modulus

$$
\frac{1}{E^{*}}=\frac{1-v_{1}^{2}}{E_{1}}+\frac{1-v_{2}^{2}}{E_{2}}
$$

This basic Hertzian theory is valid for elastic deformations and neglects surface roughness. Determined contact area is the nominal value, which can be much higher than the real contact area. Three types of components should be considered in the determination of contact resistance $R_{C}$ : constriction resistance $R_{\text {constr. }}$, bulk resistance $R_{b}$ and tunneling resistance $R_{T}$. Expression for $R_{\text {constr. }}$ of one body is:

$$
R_{\text {constr. }}=\frac{\rho}{4 a}
$$

Typical equation for bulk resistance of spherical body under elastic deformation is:

$$
\begin{aligned}
R_{b} & =\int_{-\left(r-\Delta_{2}\right)}^{r-\Delta_{1}} \rho_{1} \frac{d z}{\pi\left(r^{2}-z^{2}\right)} \\
& =\frac{\rho_{1}}{2 \pi \cdot r} \ln \left[\left(\frac{2 r}{\Delta_{1}}-1\right)\left(\frac{2 r}{\Delta_{2}}-1\right)\right]
\end{aligned}
$$

where $\rho_{1}$ is the particle resistivity and $\Delta_{1}, \Delta_{2}$ are deformations of particle. Nevertheless authors sometimes used different expressions for $R_{b}$ in the.

Tunneling resistance is related to any insulating film which may be coating of the contact surfaces, such as oxide films, or thin layer of adhesive between particles. Tunneling resistance is usually neglected in ACA modeling since it is assumed that metal to metal contact is achieved during the assembly process. Nevertheless this is not quite true for whole range of bonding pressure. Basic evaluation of tunneling resistance has been already introduced by $\mathrm{R}$. Holm in 1967 [1]. This calculation of tunneling resistance $R_{T}$ is valid for insulating layers with thickness less than 5 nm:

$$
\begin{aligned}
& R_{T}=\frac{\sigma_{T}}{\pi a^{2}} \\
& \sigma_{T}=\frac{10^{-22}}{2} \frac{A^{2}}{1+A B} e^{A B} \\
& A=7,32.10^{6}\left(t-\frac{0,72}{\Phi}\right) \\
& B=1,265.10^{-6} \sqrt{\Phi-\frac{10}{t . \varepsilon_{r}}}
\end{aligned}
$$

where $\sigma_{T}$ is tunneling,,resistivity" in $\Omega . \mathrm{cm}^{2}, t$ is a layer thickness in $\AA, \Phi$ is the work function for electron to enter from the contact to the film material in $\mathrm{eV}, \varepsilon_{\mathrm{r}}$ is relative permittivity.

Different method, more complex analysis for tunneling resistance $R_{T}$, was also developed by de J.G. Simmons [2]:

$$
R_{T}=\frac{V_{i}}{I_{i}}
$$

where

$$
\begin{gathered}
I_{i}=\frac{6,2 \cdot 10^{10}}{(\Delta S)^{2}} \cdot\left\{\begin{array}{l}
\varphi_{L} \exp \left(-1,025 \Delta S \varphi_{L}^{1 / 2}\right)- \\
\left(\varphi_{L}+V_{i}\right) \exp \left[-1,025 \Delta S\left(\varphi_{L}+V_{i}\right)^{1 / 2}\right]
\end{array}\right\} \cdot A_{i} \\
\varphi_{L}=\varphi_{0}-\left[\frac{V_{i}}{(2 t)}\right]\left(S_{1}+S_{2}\right)-\left[\frac{5,75}{K \Delta S}\right] \cdot \ln \left[\frac{S_{2}\left(t-S_{1}\right)}{S_{1}\left(t-S_{2}\right)}\right] \\
S_{1}=\frac{6}{K \varphi_{0}} ; \quad \Delta S=S_{2}-S_{1}
\end{gathered}
$$


for $V_{i}<\varphi_{0}$ :

$$
S_{2}=t\left[1-\frac{46}{\left(3 \varphi_{0} K \cdot t+20-2 V_{i} K \cdot t\right)}\right]+\frac{6}{\left(K \varphi_{0}\right)}
$$

for $V_{i}>\varphi_{0}: \quad S_{2}=\frac{\left(\varphi_{0} K \cdot t-28\right)}{V_{i} K}$

where $\mathrm{K}=\varepsilon_{\mathrm{r}}, \varphi_{0}$ is potential barrier and $t$ is a layer thickness in $\AA$.

Our paper compares three basic models for electrical contact resistance $R_{C}$ prediction - models by D.J. Williams, K.N. Chiang and M.J.Yim [3]. For calculations of contact radius $a$ is used Hertzian equation (only elastic deformations). Electrical connection is provided by $N$ particles. Final evaluations of contact resistance $R_{C N}$ between conductive pads of chip and track on substrate consists of different parts:

D.J. Williams: $R_{C}=(1 / N) * R_{\text {constr. }}$

$$
R_{C N}=\frac{1}{N}\left[\frac{\rho_{1}}{\pi}\left(\frac{1}{2 a}-\frac{1}{d}\right)\right]
$$

K.N. Chiang: $R_{C}=(1 / N) * 2 * R_{\text {constr. }}$

$$
R_{C N}=\frac{1}{4 N}\left(\rho_{1}+\rho_{2}\right) \cdot \sqrt[3]{\frac{8 N E^{*}}{3 F d}}
$$

M.J.Yim: $R_{C}=(1 / N) *\left(4 * R_{\text {constr. }}+R_{b}\right)$

$$
R_{C N}=\left[\frac{1}{2 a N}\left(\rho_{1}+\rho_{2}\right)\right]+\left[\frac{\rho_{1}}{0.524 \cdot d N}\right]
$$

Surface roughness and tunneling resistance are not considered in any of the models. Results of simulations for contact resistance prediction from these models are shown in Figure1. For evaluations have bee used these parameters: elastic linear deformations; $\mathrm{N}=1$; particle $-\mathrm{Ni}, \mathrm{d}=5 \mu \mathrm{m}, \mathrm{E}_{1}=207$ $\mathrm{GPa}, \rho_{1}=6,9 \times 10^{-8} \Omega . \mathrm{m}, v_{1}=0,31$; conductive pads $\mathrm{Cu}, \mathrm{E}_{2}=115 \mathrm{GPa}, \rho_{1}=1,7 \times 10^{-8} \Omega . \mathrm{m}, v_{1}=0,36$. From graphs is evident that differences between models are significant. Nevertheless all of these relationships between bonding force and contact area include many simplifications and finally calculated contact resistance is always lower than values of real interconnections.

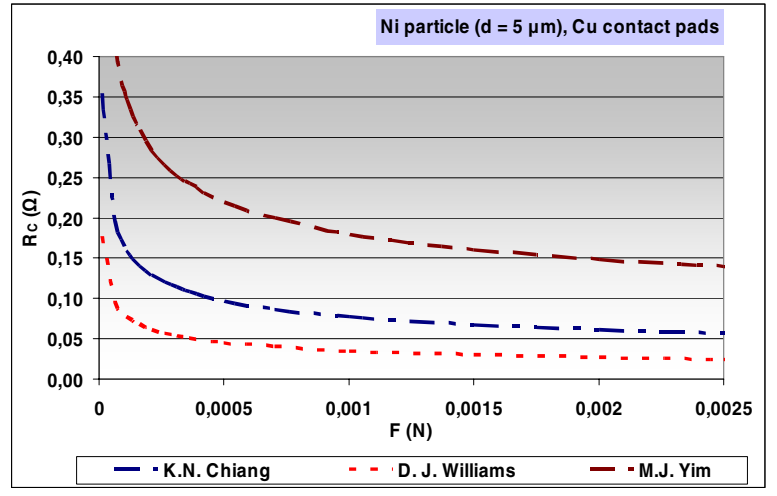

Figure 1. Predicted relationships between bonding force and electrical contact resistance $\mathrm{R}_{\mathrm{C}}$ for single particle in ACA.

Because of results from experimental measurements of interconnections resistance in ACA/ACF joints give much higher values than mentioned models, evaluations given by M.J.Yim seems to be the most exact. Therefore this model is directly compared with following experimental results and also electron tunneling effect is involves into this model.

\section{APPLIED MATERIALS AND EXPERIMENTS}

Testing samples have been prepared with assembly of SMD resistors (type 1206 0R0) with special contacts metallization $(\mathrm{CrNi}-\mathrm{Cu}-\mathrm{Ag}$ - top layer $\mathrm{Ag}$ ) for adhesive connections. The SMD resistors are not usually assembled on PCBs with ACAs, nevertheless for testing of influence of combination of curing pressure and pads metallization are these components compliant. As an adhesive has been used Loctite ACA with $\mathrm{Ni} / \mathrm{Au}$ particles, $\mathrm{d}=7$ $\mu \mathrm{m}$ and $\mathrm{T}_{\mathrm{g}}=150^{\circ} \mathrm{C}$.

Basic material of testing board is common used FR4 and material of conductive paths is plated $\mathrm{Cu} 35$ $\mu \mathrm{m}$ foil. Three different types of surface metallization of conductive paths have been used $(35 \mu \mathrm{m} \mathrm{Cu}, 35 \mu \mathrm{m}$ $\mathrm{Cu}+$ HASL (60Sn40Pb), $35 \mu \mathrm{m} \mathrm{Cu}+\mathrm{Ni} / \mathrm{Au})$. All analyses of the ACAs joints have been carried out in a range of bonding pressure $0-10 \mathrm{MPa}(0-10 \mathrm{~N} / \mathrm{bond})$ for investigation of influence of curing pressure on quality of joints. Samples were cured with hot air under defined conditions $\left(180{ }^{\circ} \mathrm{C} / 20\right.$ s). Electrical resistance of the interconnections has been measured with the LCR meter HP 4284A. The samples have been contacted with four-point probe method and measuring voltage $\sim \mathrm{V}=50 \mathrm{mV}$. Results from experimental measurements and models are shown in 
Figure 2. Simulations were performed for the same materials as in Figure1.

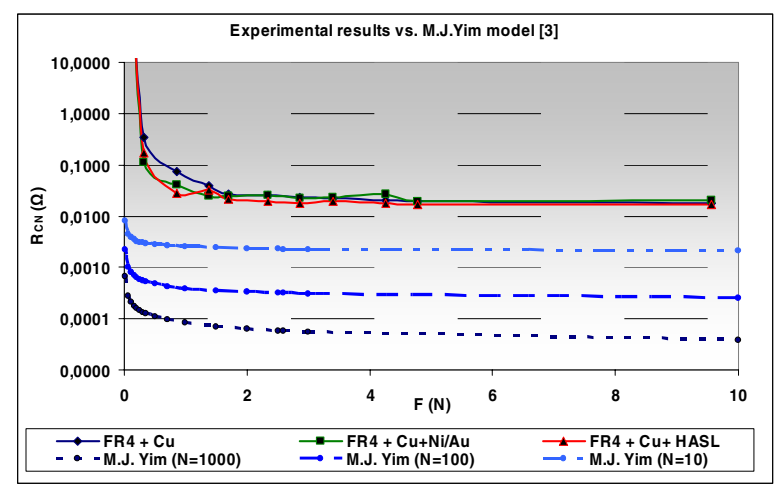

Figure 2. Comparison of experimental results and predicted electrical contact resistance $\mathrm{R}_{\mathrm{CN}}$ by M.J. Yim model without tunnelling effect $(\mathrm{N}=10 ; \mathrm{N}=100 ; \mathrm{N}=$ 1000)

From the graphs and also from the theoretical presumptions is clear that values of electrical resistance of interconnects for real testing samples are higher than predicted ones. As was already said it is due to many simplifications in models - especially neglected materials roughness and tunnelling effect and also optimal circular contact area is supposed. Therefore there is introduced extended models which include tunneling resistance for more accurate prediction of interconnection resistance.

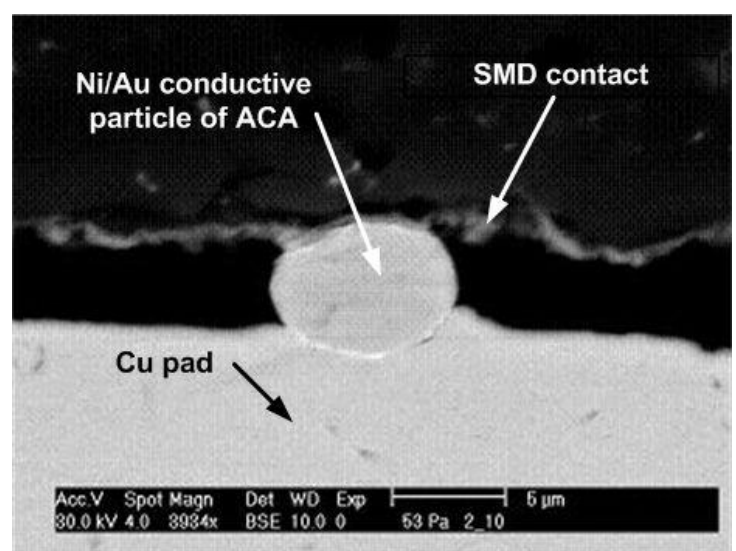

Figure 3. SEM micrograph showing ACA interconnection through $\mathrm{Ni} / \mathrm{Au}$ conductive particle under $10 \mathrm{MPa}$ pressure

Measurements and also SEM analysis show that conductive particles create good electrical interconnections between pads of component and conductive tracks on substrate. Deformation of $\mathrm{Ni} / \mathrm{Au}$ conductive particle (10 $\mathrm{MPa}$ bonding pressure) is shown in Figure 3. This deformation is low because of hard core $(\mathrm{Ni})$ of this particle. Polymer particles with high conductive layer can be deformed more and therefore initial contact resistance of these types of ACAs is lower. If zero bonding pressure is applied then the joint resistance is approximately equal to $5 \times 10^{5} \Omega$. For pressure 5-10 $\mathrm{MPa}(5-10 \mathrm{~N} /$ bond $)$ electrical resistance decreases on $16-21 \mathrm{~m} \Omega$ for all types of pad metallization. Contact area $A$ of one particle for these bonding forces and materials is around $5 \mu \mathrm{m}^{2}$.

\section{EXTENDED MODELS}

Following extended models for prediction of electrical contact resistance for anisotropic conductive adhesives combine basic model (13) and evaluations of tunneling resistance with equation (6) or (10). Both combinations are compared with experimental results. The tunneling resistance is serial-connected to the constriction and bulk resistance. Parameters for simulations: contacting area of pads $S_{1}=1 \mathrm{~mm}^{2}$; bonding pressure $10 \mathrm{~N} / \mathrm{mm}^{2} ; \mathrm{N}=500$ (1000); $\mathrm{d}=7$ $\mu \mathrm{m} ; \varepsilon_{\mathrm{r}}=4,2 ; \mathrm{t}=5,96 \AA ; \Phi=4,75 \mathrm{eV} ; \mathrm{Vi}=0,025 \mathrm{~V}$; $\mathrm{E}_{\mathrm{Au}}=77 \mathrm{GPa} ; v_{\mathrm{Au}}=0,42 ; \mathrm{E}_{\mathrm{Ni}}=207 \mathrm{GPa} ; v_{\mathrm{Ni}}=0,31$; $\mathrm{E}_{\mathrm{Cu}}=115 \mathrm{GPa} ; v_{\mathrm{Cu}}=0,36 ; \mathrm{E}_{\mathrm{Ag}}=83 \mathrm{GPa} ; v_{\mathrm{Ag}}=0,37$; $\mathrm{E}_{\mathrm{SnPb}}=30 \mathrm{GPa} ; v_{\mathrm{SnPb}}=0,40 ; \rho_{\mathrm{Au}}=2,4 \times 10^{-8} \Omega . \mathrm{m} ; \rho_{\mathrm{Ni}}$ $=6,9 \times 10^{-8} \Omega . \mathrm{m} ; \rho_{\mathrm{Cu}}=1,7 \times 10^{-8} \Omega . \mathrm{m} ; \rho_{\mathrm{Ag}}=1,5 \times 10^{-8}$ $\Omega . \mathrm{m} ; \rho_{\mathrm{SnPb}}=1,5 \times 10^{-7} \Omega . \mathrm{m}$. In extended models is involved different material composition of particles and contact pads metallization.

Tab. 1. Results from extended models - combination of M.J.Yim model - equation (13) and tunneling resistance calculation with equation (6).

\begin{tabular}{|c|c|c|c|}
\hline $\begin{array}{c}\mathbf{N}=\mathbf{5 0 0} \\
\text { Ni/Au particles }\end{array}$ & $\begin{array}{c}\mathrm{R}_{\mathrm{CN}}(\mathrm{m} \Omega) \\
(13)\end{array}$ & $\begin{array}{c}\mathrm{R}_{\mathrm{T}} \\
(\mathrm{m} \Omega) \\
(6)\end{array}$ & $\begin{array}{c}\text { Total } \\
\mathbf{R}_{\mathbf{C N}} \\
(\mathbf{m} \boldsymbol{\Omega})\end{array}$ \\
\hline \hline FR4+Cu & $8,11 \times 10^{-2}$ & 10,96 & $\mathbf{1 1 , 0 4}$ \\
\hline FR4+Cu+Ni/Au & $1,09 \times 10^{-1}$ & 13,34 & $\mathbf{1 3 , 4 5}$ \\
\hline FR4+Cu+HASL & $1,03 \times 10^{-1}$ & 7,50 & $\mathbf{7 , 6 1}$ \\
\hline
\end{tabular}

\begin{tabular}{|c|c|c|c|}
\hline $\begin{array}{c}\mathbf{N}=\mathbf{1 0 0 0} \\
\text { Ni/Au particles }\end{array}$ & $\begin{array}{c}\mathrm{R}_{\mathrm{CN}}(\mathrm{m} \Omega) \\
(13)\end{array}$ & $\begin{array}{c}\mathrm{R}_{\mathrm{T}} \\
(\mathrm{m} \Omega) \\
(6)\end{array}$ & $\begin{array}{c}\text { Total } \\
\mathbf{R}_{\mathbf{C N}} \\
(\mathbf{m} \boldsymbol{\Omega})\end{array}$ \\
\hline \hline FR4+Cu & $4,72 \times 10^{-2}$ & 9,34 & $\mathbf{9 , 3 8}$ \\
\hline FR4+Cu+Ni/Au & $6,51 \times 10^{-2}$ & 11,3 & $\mathbf{1 1 , 3 6}$ \\
\hline FR4+Cu+HASL & $6,16 \times 10^{-2}$ & 6,33 & $\mathbf{6 , 3 9}$ \\
\hline
\end{tabular}


Tab. 2. Results from extended models - combination of M.J.Yim model - equation (13) and tunneling resistance calculation with equation (10).

\begin{tabular}{|c|c|c|c|}
\hline $\begin{array}{c}\mathbf{N}=\mathbf{5 0 0} \\
\text { Ni/Au particles }\end{array}$ & $\begin{array}{c}\mathrm{R}_{\mathrm{CN}}(\mathrm{m} \Omega) \\
(13)\end{array}$ & $\begin{array}{c}\mathrm{R}_{\mathrm{T}} \\
(\mathrm{m} \Omega) \\
(10)\end{array}$ & $\begin{array}{c}\text { Total } \\
\mathbf{R}_{\mathbf{C N}} \\
(\mathbf{m} \boldsymbol{\Omega})\end{array}$ \\
\hline \hline FR4+Cu & $8,11 \times 10^{-2}$ & 5,26 & $\mathbf{5 , 3 4}$ \\
\hline FR4+Cu+Ni/Au & $1,09 \times 10^{-1}$ & 5,76 & $\mathbf{5 , 8 7}$ \\
\hline FR4+Cu+HASL & $1,03 \times 10^{-1}$ & 4,34 & $\mathbf{4 , 4 4}$ \\
\hline
\end{tabular}

\begin{tabular}{|c|c|c|c|}
\hline $\begin{array}{c}\mathbf{N}=\mathbf{1 0 0 0} \\
\text { Ni/Au particles }\end{array}$ & $\begin{array}{c}\mathrm{R}_{\mathrm{CN}}(\mathrm{m} \Omega) \\
(13)\end{array}$ & $\begin{array}{c}\mathrm{R}_{\mathrm{T}} \\
(\mathrm{m} \Omega) \\
(10)\end{array}$ & $\begin{array}{c}\text { Total } \\
\mathbf{R}_{\mathbf{C N}} \\
(\mathbf{m} \boldsymbol{\Omega})\end{array}$ \\
\hline \hline FR4+Cu & $4,72 \times 10^{-2}$ & 3,44 & $\mathbf{3 , 4 8}$ \\
\hline FR4+Cu+Ni/Au & $6,51 \times 10^{-2}$ & 3,76 & $\mathbf{3 , 8 2}$ \\
\hline FR4+Cu+HASL & $6,16 \times 10^{-2}$ & 2,83 & $\mathbf{2 , 8 9}$ \\
\hline
\end{tabular}

Tab. 3. Experimental results of contact resistance measurements for bonding pressure $10 \mathrm{~N} /$ bond

\begin{tabular}{|c|c|}
\hline $\begin{array}{c}\text { Pads } \\
\text { metallization }\end{array}$ & $\begin{array}{c}\text { Contact resistance } \\
\mathbf{R}_{\mathbf{C N}} \\
(\mathbf{m} \boldsymbol{\Omega})\end{array}$ \\
\hline \hline FR4+Cu & $\mathbf{1 7 , 9 7}$ \\
\hline FR4+Cu+Ni/Au & $\mathbf{2 0 , 6 9}$ \\
\hline FR4+Cu+HASL & $\mathbf{1 6 , 9 3}$ \\
\hline
\end{tabular}

Extended models where is not neglected tunneling effect in thin insulating layer between particles and pads show higher values of electrical contact resistance than simplified models. Tunneling resistance part is dominant and increases total resistance of interconnection $R_{C}$. From the results in Table1, Table 2 and Table 3 is evident that extended model which combine basic M.J.Yim model (equation 13) and easier evaluation of tunneling resistance by $R$. Holm theory (equation 6) provides more accurate approaching to the real values of total contact resistance $R_{C N}$. Nevertheless main limitations of this model is using constant thickness of thin insulating layer, involving only elastic deformations in materials and neglected material roughness. Final equation is:

$R_{C N}=\frac{1}{N} \cdot\left\{\begin{array}{l}\frac{\rho_{1}+\rho_{2}}{4 a_{1}}+\frac{\rho_{1}+\rho_{3}}{4 a_{2}}+\frac{\rho_{1}}{0.524 d} \\ +\left(\frac{1}{a_{1}^{2}}+\frac{1}{a_{2}^{2}}\right) \cdot\left(\frac{10^{-22}}{2 \cdot \pi} \cdot \frac{A^{2}}{1+A \cdot B} \cdot e^{A \cdot B}\right)\end{array}\right\}$ where $\rho_{1}, \rho_{2}, \rho_{3}$ is resistivity of particle, component pads and substrate pads, respectively, $a_{1}, a_{2}$ is a contact radius between particle and component pads, particle and substrate pads, respectively.

\section{Conclusion}

This study models the electrical contact resistance prediction for anisotropic conductive adhesives. Introduced extended models consider the possible existence of an ultra-thin insulating layer between the particles and contacting surfaces. For the electrical current which flow across this insulating film the electrons must tunnel through the energy barrier imposed by the film. This electrical tunneling resistance $R_{T}$ is much higher than the constriction resistance $R_{\text {constr. }}$ and the bulk resistance $R_{b}$ of the particle. Therefore extended models, especially equation (14), which include tunneling resistance, provide more accurate approaching to the real values of total contact resistance of the interconnections in the ACAs/ACFs.

\section{ACKNOWLEDGMENTS}

This research was supported by grant MSM no. 6840770021 - Diagnostic of Materials, Czech Republic.

\section{REFERENCES}

[1] Holm, R.: Electric Contacts, New York: Springer, 1967.

[2] Simmons, J.G., "Generalized formula for the electric tunnel effect between similar electrodes separated by a thin insulating film", Journal of Applied Physics, Vol. 34, Issue 4, 1963, pp. 1793-1803.

[3] Yim, M.J., Paik, K.W., "Design and Understanding of Anisotropic Conductive Films (ACF's) for LCD Packaging", IEEE Transaction on Components, Packaging, and Manufacturing Technology A, vol.21, no.2, June 1998, pp. 226-234.

[4] Duraj, A., Mach, P., "Diagnostic Tools for Evaluation of the Quality of the Anisotropic Conductive Adhesive Joints", in Proc. $29^{\text {th }}$ Int. Spring Sem. Electronics Technology (ISSE'06), St. Marienthal, Germany, May 10-14, 2006, pp. 118-119.

[5] Jackson, R.L.., Kogut, L., "Electrical Contact Resistance Theory for Anisotropic Conductive Films Considering Electron Tunnelling and Particle Flattening", IEEE Transaction on Comp. and Packaging Technologies, vol.30, Issue 1, March 2007, pp. 59-66. 